\title{
Aspects microscopiques de l'endommagement par érosion de cavitation de métaux et alliages utilisables en construction navale
}

\author{
J. L. Heuzé \\ Service technique des constructions et armes navales, Paris
}

\section{Introduction}

\begin{abstract}
Afin de mieux comprendre les phénomènes d'érosion survenant sur les pièces en service soumises à la cavitation (pales d'hélice, corps de pompe, aubes de turbine, etc.), une étude a été entreprise visant à mettre en évidence les modifications de la structure au cours de l'endommagement, proposer des paramètres permettant de quantifier ce dommage, et mieux comprendre les mécanismes relatifs à ce type de dégradation.

On rappelle que dans un écoulement liquide, la cavitation est la conséquence de l'apparition de cavités remplies de vapeur ou de gaz, puis de leur implosion sous l'effet d'une brusque augmentation de pression. Quant à la formation des cavités, elle est due à une diminution locale de la pression de telle manière que celle-ci devienne inférieure à la tension de vapeur du liquide.
\end{abstract}

\section{Dispositif utilisé pour simuler la cavitation}

\subsection{Description de la veine tourbillon NEYRTEC [1]}

Cette veine tourbillon (figs. 1 et 2 ) est constituée d'un circuit hydraulique comprenant : une cuve haute pression (repère 1 ), un obturateur (repère 2 ), la veine tourbillon proprement dite (repère 3), une cuve basse pression (repère 4), une pompe de refoulement (repère 5), un moteur entraînant l'obturateur (repère 7), une petite cuve annexe (repère 6) permet par ailleurs, le dégazage du liquide.

\subsection{Principe de fonctionnement de la veine tourbillon}

La veine tourbillon est caractérisée par une arrivée tangentielle du liquide et une sortie axiale (fig. 1).

Microscopic aspects of damage caused by cavitation erosion of metals and alloys able to be used for shipbuilding.

A study relating to the cavitation erosion phenomenon has been carried out with the help of an eddy vein, on 22 grade pure copper, CuZn 20 brass, two grades of monophased cupro-aluminium, CuAl 2 and CuAl 6 , as well as on austenitic stainless steel, grade Z2 CND 17-12.

During the first stage known as the incubation period, damage occurred by deformation of the target. This deformation is directly connected with the intrinsic properties of the material (structure, stacking defect energy).

The second stage is the actual erosion stage which leads to a loss of cohesion as well as the fragmentation 


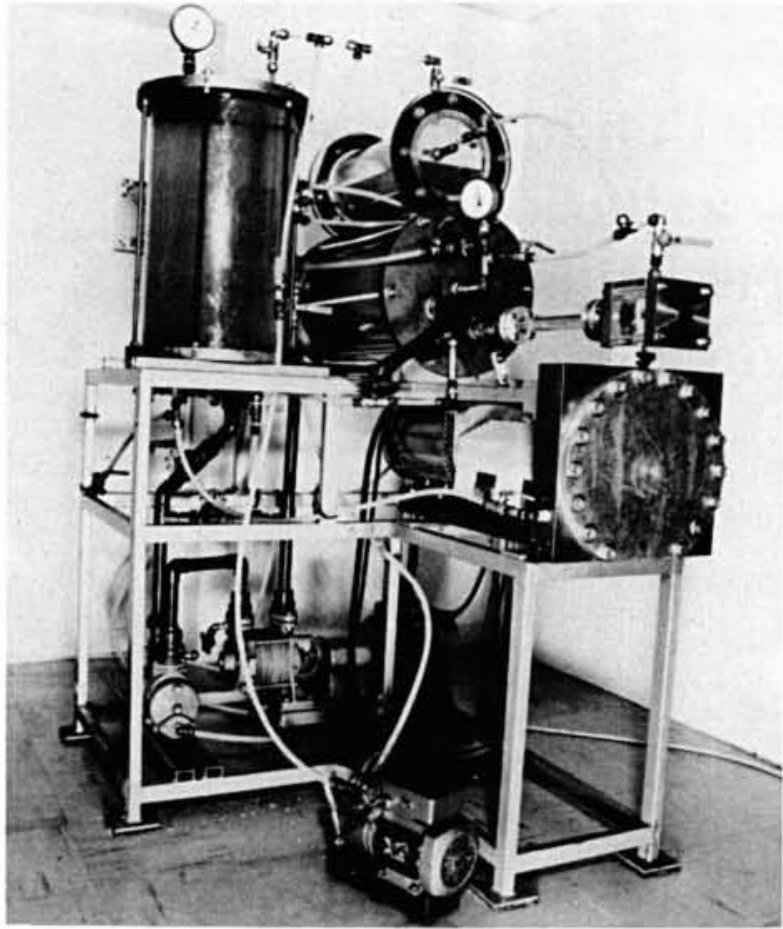

1. Veine Tourbillon Neyrtec. Vue d'ensemble du dispositif.

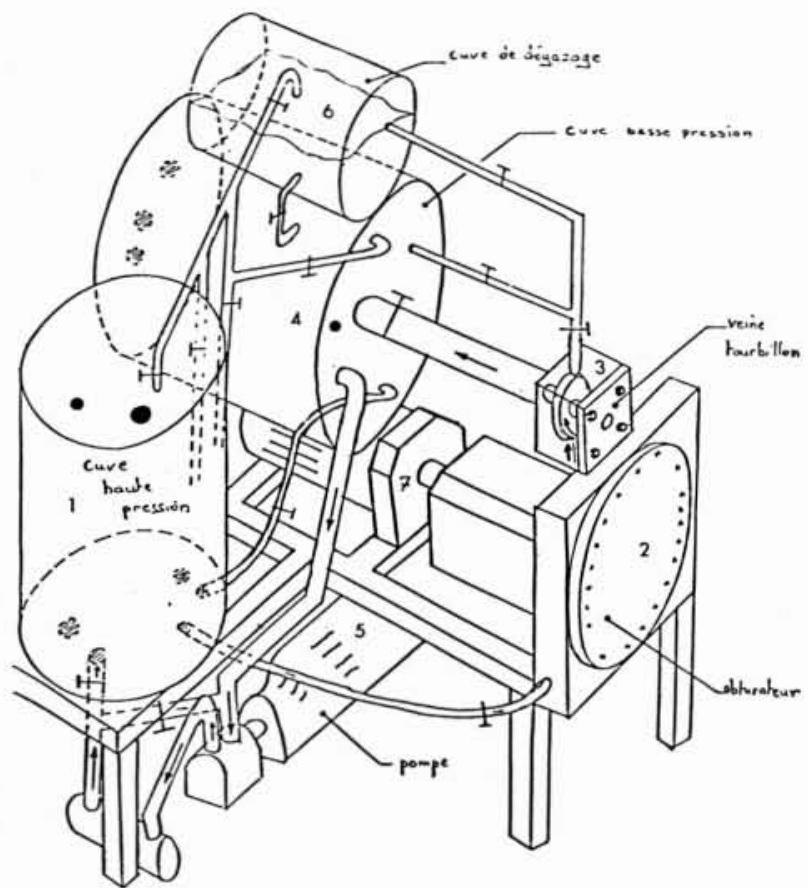

2. Schéma de principe de la Veine Tourbillon Neyrtec.
A l'aide de l'obturateur tournant, on provoque un arrêt brutal de l'alimentation de la veine, ce qui génère une onde de dépression et la formation d'une cavité de vapeur au centre de la veine, puisque c'est dans cette zone que les vitesses circonférentielles sont les plus grandes donc les pressions les plus faibles.

L'onde de dépression ainsi générée se propage dans la canalisation aval pour venir se réfléchir sur le liquide de la cuve basse pression. Cette onde revient ensuite dans la veine sous forme d'une onde de pression. Elle entraîne la cavité de vapeur dans le conduit conique et provoque son implosion (collapse) au niveau de la surface de l'échantillon situé à l'extrémité de ce conduit.

\subsection{Conditions expérimentales}

Les conditions expérimentales sont les suivantes :

$\begin{array}{ll}\text { Pression amont } & : 0,5 \mathrm{MPa}(0,5 \text { bars }) \\ \text { Pression aval } & : \text { Pression atmosphérique } \\ \text { Débit } & : 30 \mathrm{l} / \mathrm{min}\end{array}$

Milieu d'essai : Eau déminéralisée, dégazée, additionnée d'inhibiteur de corrosion : $\quad\left(\mathrm{Na}_{2} \mathrm{Cr}_{2} \mathrm{O}_{7}\right.$ : $2,5 \mathrm{~g} / \mathrm{l})$ tamponnée avec $\left(\mathrm{Na}_{3} \mathrm{PO}_{4}, 12 \mathrm{H}_{2} \mathrm{O}: 2,0 \mathrm{~g} / \mathrm{l}\right)$

$\mathrm{pH}$ de la solution : $6,7 \pm 0,2$

Teneur moyenne en oxygène de la solution :

$$
\begin{aligned}
& : 0,5 \pm 0,2 \mathrm{ppm} \\
& \left(0,5 \pm 0,2 \mathrm{mg} \cdot \mathrm{1}^{-1}\right)
\end{aligned}
$$

Température : $25 \pm 3{ }^{\circ} \mathrm{C}$

Cadence $\quad:$ 7,2 impacts par seconde

Surface moyenne impactée : $S \# 0,16 \pm 0,4 \mathrm{~cm}^{2}$.

\section{Matériaux étudiés}

Les métaux et alliages étudiés ont tous une structure cubique à faces centrées. Leur composition chimique fait l'objet du tableau 1.

Un traitement thermique, tableau 2, a été effectué sur les cibles de cavitation après usinage, afin, d'une part, de disposer d'un état de référence parfaitement défini exempt de tout écrouissage, et, d'autre part, d'une taille de grain similaire. Cette taille de grain est voisine de $0,2 \mathrm{~mm}$ pour tous les matériaux. De plus, la surface soumise aux impacts de cavitation a été polie électrolytiquement. Le tableau 2 regroupe, pour chacun des matériaux, outre les conditions de traitement thermique, la dureté initiale relevée sur les pastilles juste avant cavitation et la valeur de leur énergie de défaut d'empilement $\left(\gamma_{s}\right)$ relevée dans la littérature.

\section{Résultats expérimentaux}

L'objectif de l'étude étant de mettre en évidence, puis de tenter de qualifier et quantifier l'endommagement, les méthodes d'investigation retenues sont les suivantes :

- mesures de perte de volume qui donnent une idée précise du degré de résistance des différents métaux et alliages à l'érosion de cavitation, on accède ainsi aux vitesses d'érosion de ces matériaux ;

- observations en microscopie optique, en microscopies électronique à balayage et en transmission pour déterminer les modes de déformation; 
EiQ. 3e: COURBES DE PERIE DE YOLUME

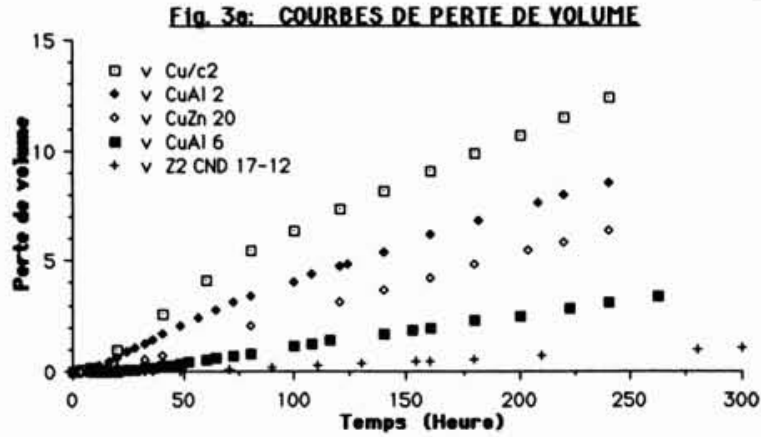

Ele. 3b: COURBES D'EROSION

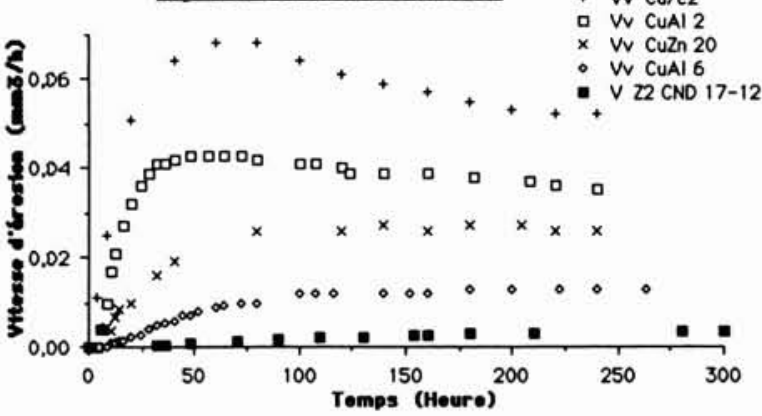

3.

- mesures de microdureté sur coupe pour évaluer la profondeur de la zone plastifiée.

\subsection{Courbes d'érosion}

Afin de quantifier l'érosion occasionnée par la cavitation,

2 types de courbes sont présentées :

D'une part: $\Delta v=f(t)$ appelée courbe de perte de volume (fig. $3 a$ ).

D'autre part: $V=g(t)$ considérée comme la courbe d'érosion proprement dite (fig. $3 b$ )

où : $t$ est le temps en heure,

$\Delta v$ est la perte de volume en $\mathrm{mm}^{3}$,

$V$ est la vitesse d'érosion en $\mathrm{mm}^{3} / \mathrm{h}$.

Les courbes de perte de volume permettent d'évaluer globablement la résistance des matériaux à la cavitation et d'en établir une première, mais grossière classification. Ainsi, pour les cinq matériaux étudiés, cette classification par ordre de résistance croissante à l'érosion de cavitation est la suivante :

\section{$\mathrm{Cu} / \mathrm{c} 2<\mathrm{CuAl} 2<\mathrm{CuZn} 20<\mathrm{CuAl} 6<\mathrm{Z} 2 \mathrm{CND} 17-12$}

Les courbes d'érosion permettent, par la détermination des paramètres caractéristiques $T_{i}, T_{m}$, et $V_{m}$, d'affiner les résultats obtenus grâce aux courbes de perte de volume.

$T_{i}$ est la durée de la période d'incubation.

$T_{m}$ est la durée nécessaire pour atteindre la vitesse d'érosion maximale du matériau.

$V_{m}$ est la vitesse d'érosion maximale.

Les valeurs de ces paramètres, pour chacun des matériaux testés sont regroupées dans le tableau 3.

\section{Tableau 1}

Composition chimique des cibles.

\begin{tabular}{|c|c|c|c|c|c|c|c|c|c|c|c|c|}
\hline Eléments & $\mathrm{Cu}$ & Al & $\mathrm{Fe}$ & Ag & $\mathrm{Mn}$ & $\mathrm{Ni}$ & $\mathrm{O}_{2}$ & P & $\mathrm{Pb}$ & $S$ & $\mathrm{Si}$ & $\mathrm{N}_{2}$ \\
\hline $\mathrm{Cu} / \mathrm{c} 2$ & 99.99 & - & - & 0,0025 & - & - & 0,0019 & 0,001 & 0,0015 & 0,002 & - & - \\
\hline CUAI 2 & 97,71 & 2,08 & 0.044 & - & 0.002 & 0,009 & 0,0020 & - & 0.016 & 0,0017 & 0,040 & $(0.001$ \\
\hline CuAl 6 & 94.20 & 5.75 & 0.030 & - & 0.001 & 0,006 & 0.0020 & - & 0.004 & 0.0007 & 0.019 & $<0.001$ \\
\hline
\end{tabular}

\begin{tabular}{|c|c|c|c|c|c|c|c|c|c|c|}
\hline Eléments & Cu & Al & Zn & Fe & P & PD & S & Si & Sn & Remarque \\
\hline CuZn 20 & 80,95 & ND & 19,04 & ND & ND & ND & - & ND & 0,01 & ND = Non. Détecté \\
\hline
\end{tabular}

\begin{tabular}{|l|l|c|c|c|c|c|c|c|c|c|c|}
\hline Elèments & Fe & C & Cr & Mn & Mo & Ni & $P$ & S & Si & $\mathrm{N}_{2}$ & Remarque \\
\hline 22CND 17/12 & Reste & 0,026 & 18,03 & 1,37 & 2,47 & 13,9 & 0,01 & 0,0065 & 0,56 & - & - Non mesuré \\
\hline
\end{tabular}

Tableau 2

Traitement thermique et Caractéristiques (dureté et énergie de défaut d'empilement).

\begin{tabular}{|c|c|c|c|c|}
\hline MATERIAUX & TRAITEMENTS THERMIQUES & Dureté init. $(\mathrm{H} v)$ & $r_{s}(m / m-2)$ & Rǘärences \\
\hline $\mathrm{Cu} / \mathrm{c} 2$ & Recult sous vide $30 \mathrm{~min}$ a $750^{\circ} \mathrm{C}$ & $54 \pm 5$ & $70 \pm 15$ & {$[2] \Leftrightarrow[7]$} \\
\hline CUAI 2 & Recuit sous argon theure aे $700^{\circ} \mathrm{C}$ & $56 \pm 5$ & $32 \pm 4$ & [2] et [5] \\
\hline CuAl 6 & Recult sous argon 1heure \& $700^{\circ} \mathrm{C}$ & $87 \pm 5$ & $8 \pm 2$ & [2] et [5] \\
\hline cuzn 20 & Recult sous argon $30 \mathrm{~min} .6700^{\circ} \mathrm{C}$ & $90 \pm 5$ & $22 \pm 4$ & {$[2][3][7]$} \\
\hline $22 \mathrm{CND} 17.12$ & Maintien $4 \mathrm{~h}$ d $1000^{\circ} \mathrm{C} / \mathrm{Hypertrempe}$ & $160 \pm 5$ & $25 \pm 5$ & [8] ot [9] \\
\hline
\end{tabular}

Tableau 3

Paramètres caractéristiques et coefficient de corrélation.

\begin{tabular}{|c|c|c|c|c|c|c|}
\hline MATERIAUX & ${ }^{11}$ (heure) & $\mathrm{K}_{\mathrm{Ti}}$ & Im (heure) & $\mathrm{K}_{\mathrm{Im}}$ & $V m_{(m m 3 / n)}$ & $\mathrm{k}_{\mathrm{rm}}$ \\
\hline $\mathrm{Cu} / \mathrm{c} 2$ & 3 & 1 & 60 & 1 & 0,068 & 1 \\
\hline CUAI 2 & 6 & 2 & 64 & 1.10 & 0,043 & 1,58 \\
\hline Cuzn 20 & 8 & 2,67 & 140 & 2,33 & 0,026 & 2,62 \\
\hline CUAl 6 & 16 & 5,33 & 240 & 4,00 & 0,013 & 5,23 \\
\hline 22 CND 17.12 & 30 & 10 & 600 & 10 & 0,0047 & 14,46 \\
\hline
\end{tabular}



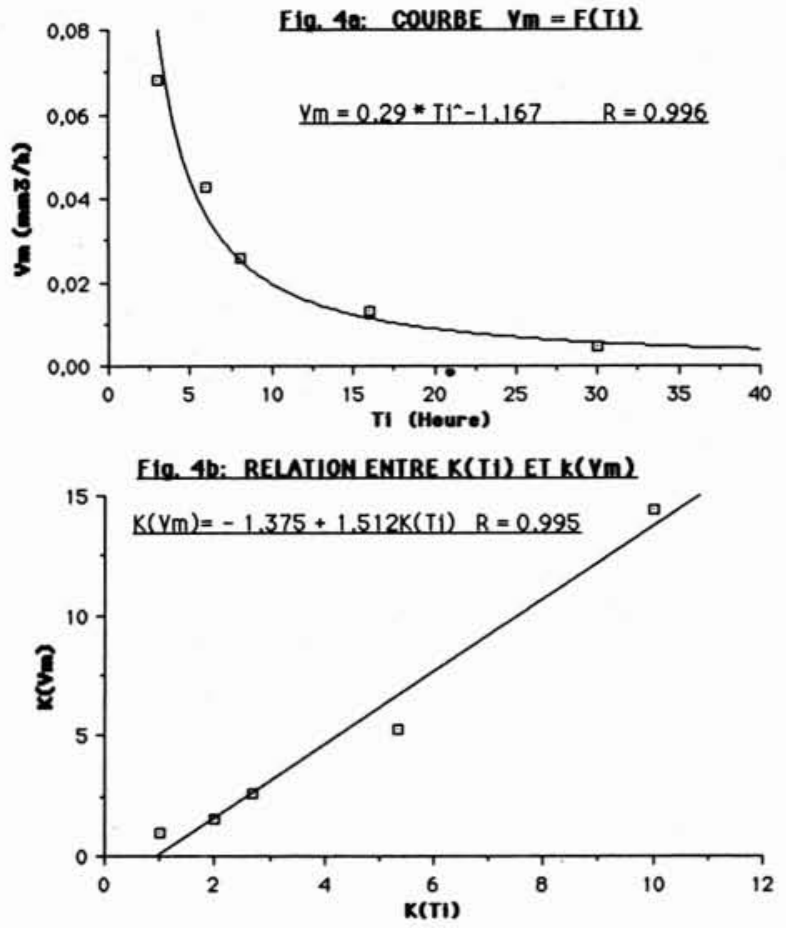

4.

5. Examen de surface au MEB.

6. Examen sur coupe transversale.

7. Examen sur lames minces au MET
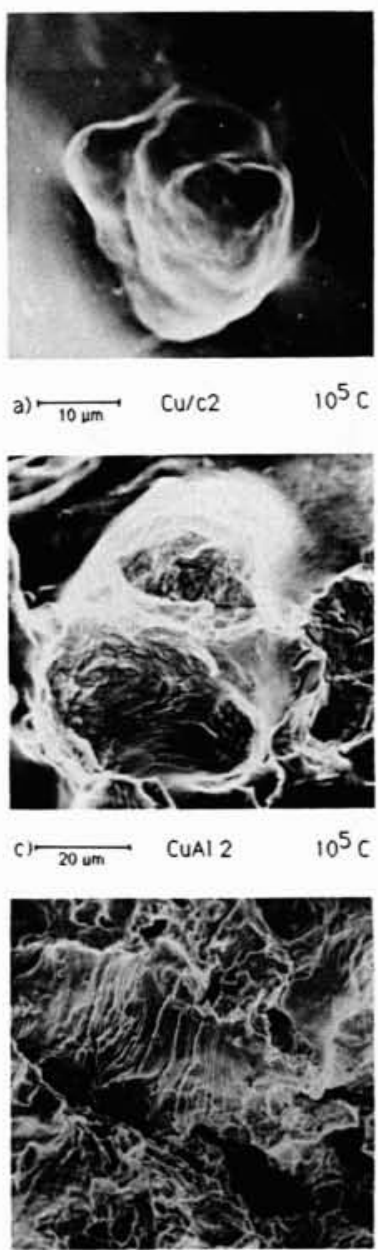

e) $\frac{25 \mathrm{um}}{\mathrm{Cu}} \quad \mathrm{Cun} 20 \quad 5 \cdot 10^{5} \mathrm{C}$
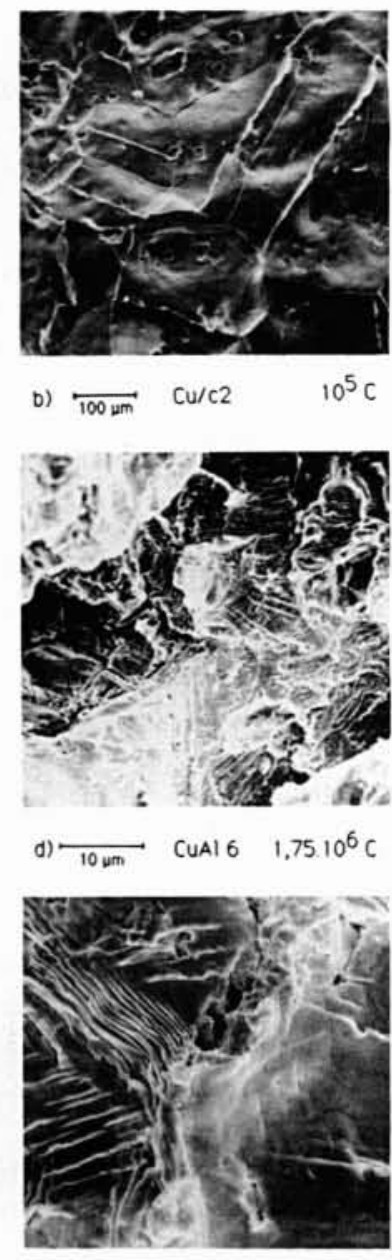

f) $\underset{20 \mathrm{um}}{2} 22 \mathrm{CND} 17-12 \quad 10^{6} \mathrm{C}$

5.

Le tracé de la courbe $V_{m}=f\left(T_{i}\right)$ permet de mettre en évidence la relation suivante:

$$
V_{m}=0,29 * T_{i}^{-1,167}
$$

Le coefficient de corrélation obtenu : $R=0,996$, montre que les points expérimentaux sont très proches de la courbe théorique. Toutefois, nous ne nous sommes adressés qu'à un nombre restreint de matériaux et il serait souhaitable, dans l'avenir, de contrôler la validité de la relation sur une plus large gamme de métaux et alliages.

Afin d'affiner les résultats obtenus, nous avons calculé, pour chacun de ces trois paramètres, un « coefficient de corrélation " $K$ en choisissant le cuivre comme métal de référence. Les valeurs obtenues sont regroupées dans le tableau 3.

Les coefficients de corrélation $K$ ont été déterminés de la façon suivante :

$$
\begin{aligned}
K_{T_{i}} & \left.=T_{i}(\text { matériau }) / T_{i} \text { (cuivre }\right) \\
K_{T_{m}} & =T_{m}(\text { matériau }) / T_{m}(\text { cuivre }) \\
K_{V_{m}} & =V_{m}(\text { cuivre }) / V_{m}(\text { matériau })
\end{aligned}
$$

( $K_{V_{m}}$ est le rapport inverse des deux précédents afin de rester homogène avec ces derniers.)

L'analyse des valeurs obtenues permet de constater : une bonne similitude pour les alliages cuivreux entre $K_{T_{i}}$ et $K_{V_{m}}$, et, pour l'acier étudié, $K_{V_{m}}>K_{T_{i}}$.

Nous avons donc tracé, pour les alliages cuivreux, la droite : $K_{V_{m}}=f\left(K_{T_{i}}\right)$ (fig. $\left.4 b\right)$. Elle a pour équation : $K_{V_{m}}=1,006 K_{T_{i}}-0,159$ et pour coefficient de corrélation $R=0,995$. Cette relation, très proche de $K_{V_{m}}=$ $K_{T_{i}}$ est, d'un point de vue industriel, fort intéressante car elle permet par la simple connaissance de la durée de la période d'incubation d'accéder rapidement et sans calcul à la vitesse d'érosion maximale.

\subsection{Examen au microscope électronique à balayage}

Les examens MEB effectués sur les 5 métaux et alliages permettent d'observer que :

1. Sur les métaux ductiles comme le cuivre et ses alliages, l'endommagement procède, au début de la période d'incubation d'une part, par la formation de 

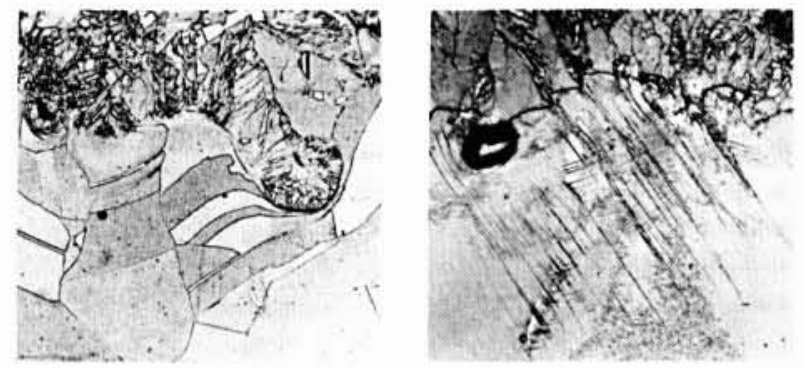

a) $\times 400 \quad$ Cu/c2 $\quad 10^{5} \mathrm{C} \quad \stackrel{25 \mathrm{~mm}}{\longrightarrow}$ D) $510^{5} \mathrm{C} \quad \mathrm{CuZn} 20 \quad \times 400$
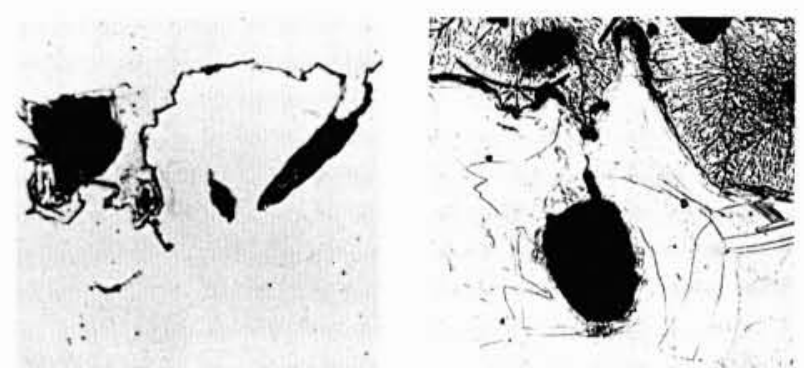

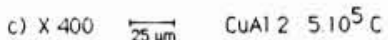

d) 25

$\times 400$
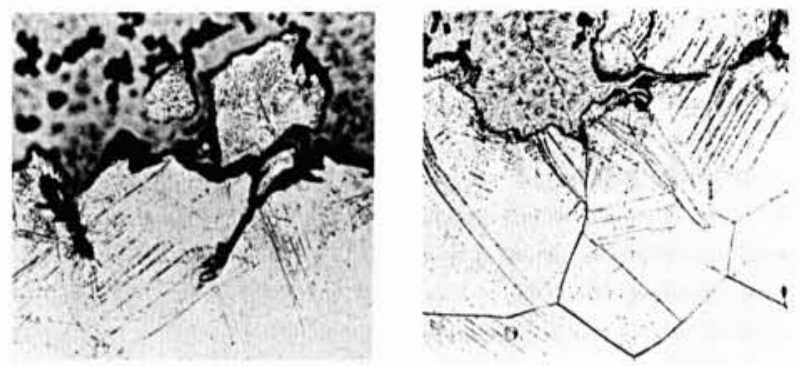

e) $\times 400 \quad 2$

CUAl $61,75 \cdot 10^{6} \mathrm{C}$ f) $\overrightarrow{25 \mathrm{~mm}}$

$\times 400$

6.

petites cavités de 20 à $30 \mu \mathrm{m}$ de diamètre avec formation d'un bourrelet de métal repoussé (fig. $5 a$ ), et d'autre part, par une ondulation générale de la surface et une délinéation des joints de grains et de macles (fig. $5 b$ ). En fin de période d'incubation, la superposition des petites cavités jointe à l'ondulation générale de la surface favorisent l'apparition de cratères de dimensions beaucoup plus importantes que ces cavités puisqu'ils peuvent atteindre de 80 à $200 \mu \mathrm{m}$ de diamètre. Le fond et les parois des cavités et des cratères sont tapissés d'intrusions et d'extrusions dont l'aspect apparaît fort similaire à des stries de fatigue ( $f i g .5 c$ et $d$ ).

De plus, on observe également l'apparition de petites fissures transgranulaires de 5 à $10 \mu \mathrm{m}$ de diamètre qui se développent selon plusieurs directions et qui conduisent à la fragmentation des grains au fond des cavités (fig. $5 d$ ). Des fissures se développent également au niveau des joints de grains provoquant des décohésions intergranulaires (fig. $5 d$ et f). On notera enfin, que les stries observées à l'intérieur des cavités sont plus sinueuses dans le $\mathrm{Cu} / \mathrm{c} 2$ et le $\mathrm{CuAl} 2$ (fig. $5 c$ ) que dans le $\mathrm{CuAl} 6$ (fig. $5 d$ ) où elles sont bien parallèles.
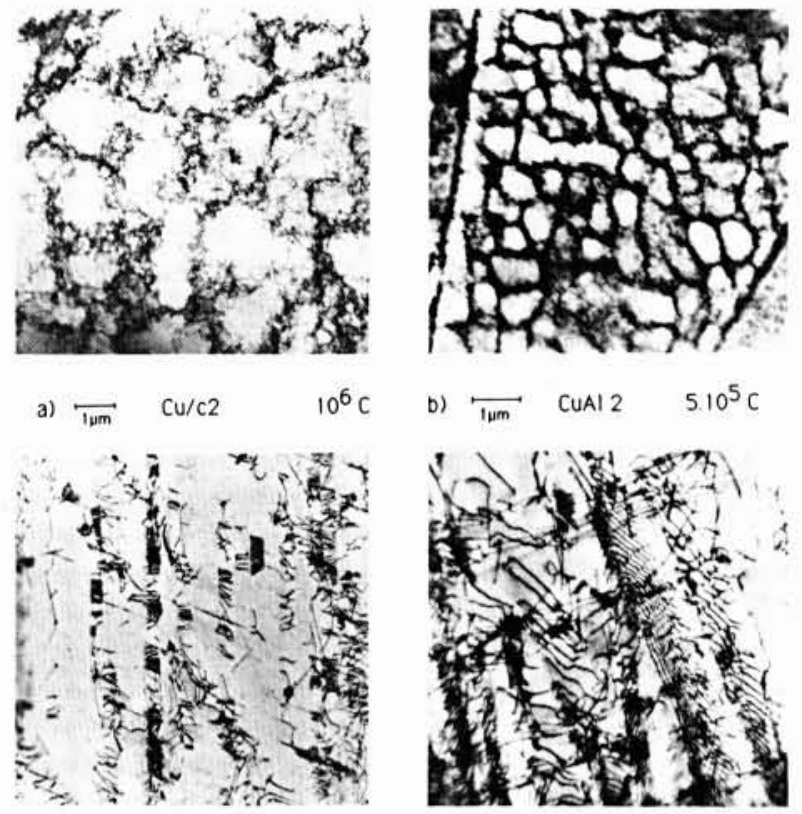

b) $\stackrel{T}{\mathrm{Tm}} \mathrm{CUAI} 2 \quad 5.10^{5} \mathrm{C}$

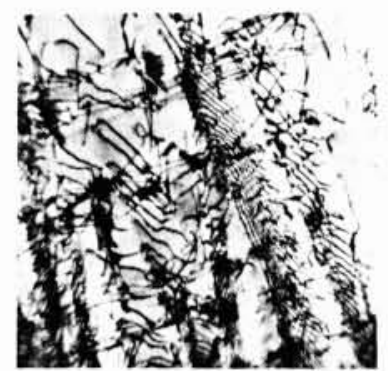

c) $\stackrel{\longrightarrow}{0.14 \mathrm{~m}}$ CUAI2 $2,5 \quad 10^{6} \mathrm{C}$
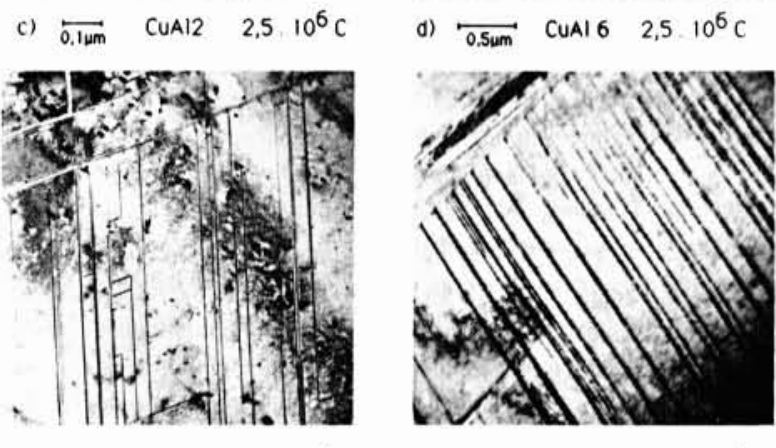

e) $\overrightarrow{0.1 \mathrm{um}}$ CuZn $5 \cdot 10^{4} \mathrm{C}$

f) $\underset{0.5 \mathrm{~mm}}{2} 22 \mathrm{CND} 17-12 \quad 5 \cdot 10^{5} \mathrm{C}$

7.

2. Sur l'acier inoxydable, on observe durant la phase d'accélération (érosion) des bandes de déformation très prononcées selon 2 directions formant un angle d'environ $60^{\circ}$. Ces bandes de déformation sont constituées de lignes de glissement persistantes et parallèles qui forment un réseau d'intrusions/extrusions très marqués, conduisant localement à des concentrations de contraintes importantes qui constituent des sites de germination privilégiés des microfissures.

\subsection{Examen sur coupe transversale}

Les examens sur coupe ont été pratiqués après un dépôt électrolytique, sur la surface cavitée, de cuivre pour les cibles en cuivre et en laiton, de nickel pour celles en cupro-aluminium, et de fer pour celles en acier inoxydable.

On observe tout d'abord que la progression des cavités s'effectue rarement selon la normale à la surface impactée. Elles forment plutôt un angle proche de $45^{\circ}$ avec la surface de la cible (fig. $6 a, c, e$ ). Les cavités progressent indifféremment vers la droite ou vers la gauche (fig. $6 a, c$ 

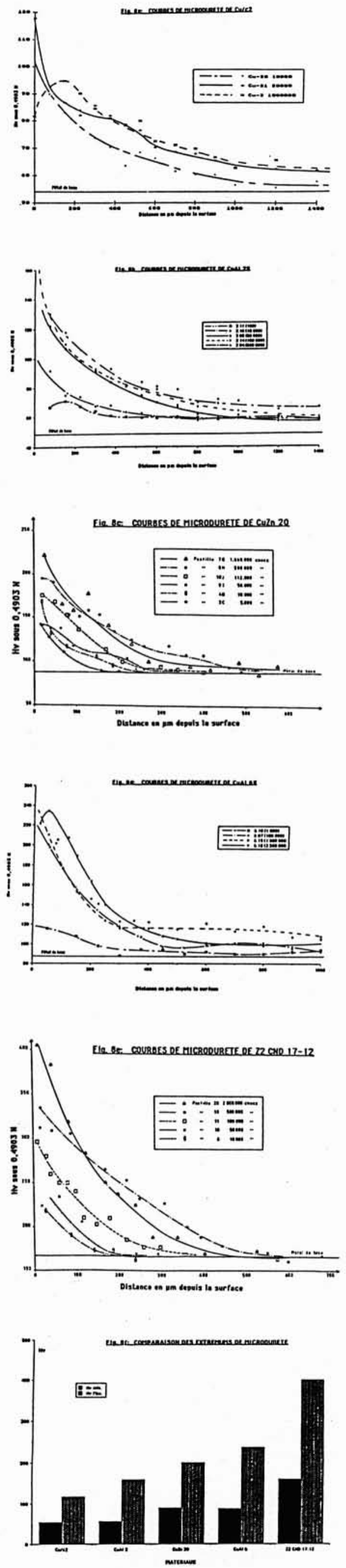

et e). On observe également la présence de cavités qui ne débouchent pas en surface, à l'endroit où la coupe a été effectuée (fig. $6 c$ et $d$ ), ce qui confirme que certaines cavités ne se propagent pas orthogonalement à la surface mais bien de manière inclinée dans plusieurs directions. Sous les cavités, les déformations sont très intenses ( $f i g$. $6 a)$. Dans le cuivre, les macles de croissance (recuit) sont très déformées et suivent le profil de la cavité. On notera également la présence de lignes de glissement persistantes autour des cavités.

Sur le laiton (fig. $6 b$ ), outre les observations précédentes, on relève la présence d'extrusions au niveau des macles mécaniques produites par la déformation (ondes de chocs). Ces extrusions, comme on l'a déjà signalé au paragraphe précédent, servent d'amorce à des microfissures. Lorsque l'on atteint la vitesse d'érosion maximale, on note sur le $\mathrm{CuAl} 6$ (fig. $6 e$ et $f$ ) la présence de petites fissures de 4 à $6 \mu \mathrm{m}$ de longueur qui se propagent selon un mode transgranulaire en convergent. On observe également des décohésions aux joints de grains (fig. $6 f$ ). On note enfin, comme sur le laiton, la présence de macles mécaniques et de lignes de glissement persistantes indiquant que l'on a affaire à une déformation plastique importante.

\subsection{Courbes de microdureté}

Les courbes de microdureté sur coupe transversale effectuées sur les 5 matériaux étudiés sont regroupées dans la figure 8 . Les valeurs remarquables obtenues ont été consignées dans le tableau 4. L'analyse de ces valeurs permet de constater :

- une augmentation importante de la dureté sous la surface de la zone cavitée pour tous les matériaux testés (écrouissage) ;

- que le taux d'écrouissage représenté par $H V \max / H V \min$ (colonne 4) varie peu : entre 2,2 et 2,8 suivant les matériaux ;

- une zone plastifiée environ 2 fois plus profonde pour le $\mathrm{Cu} / \mathrm{c} 2$ et le $\mathrm{CuAl} 2$ que pour les trois autres alliages; - une durée, pour obtenir $H V$ max, environ 10 à 20 fois plus courte pour le $\mathrm{Cu} / \mathrm{c} 2$ et le $\mathrm{CuAl} 2$ que pour le $\mathrm{CuAl}$ 6, le CuZn20 et le Z2 CND 17-12.

\section{Tableau 4}

Résultats des essais de microdureté.

\begin{tabular}{|c|c|c|c|c|c|c|}
\hline MATERIAUX & $\mathrm{Hr}_{\text {min }}$ & $\mathrm{HV}_{\text {Max }}$ & $\mathrm{Hv}_{\text {Max }} / \mathrm{Hv}_{\min }$ & $T_{\text {HVMex }}(\mathrm{h})$ & ) Profondeur ${ }_{(\mu \mathrm{m})}$ & $\gamma_{s}\left(\mathrm{mum}^{-2}\right)$ \\
\hline $\mathrm{cu/c2}$ & 54 & 118 & 2,19 & 2 & 1200 & 70 \\
\hline CuAl 2 & 56 & 159 & 2,84 & 4 & 1000 & 32 \\
\hline cuzn 20 & 90 & 200 & 2.22 & 60 & 450 & 22 \\
\hline CuAl 6 & 87 & 236 & 2,71 & 40 & 600 & 8 \\
\hline 22 CND 17.12 & 160 & 406 & 2,54 & 80 & 500 & 25 \\
\hline
\end{tabular}

8. 


\subsection{Examen au microscope électronique à transmission}

Les lames minces ont été prélevées directement sous la surface soumise à la cavitation.

Pour le $\mathrm{Cu} / \mathrm{c} 2$ et le $\mathrm{CuAl} 2$ (fig. $7 a$ et $b$ ), le mécanisme de déformation mis en évidence est le suivant : Arrangement des dislocations en un réseau tridimensionnel de cellules, caractéristique du glissement dévié des dislocations vis. Le maclage mécanique n'a pas été mis en évidence.

Pour le $\mathrm{CuAl}$ 6, le CuZn20 et l'acier Z2 CND 17-12, la déformation s'opère par glissements planaires (fig. $7 c$ et d) avec formation de défauts d'empilement ( $f i g .7 e$ ) puis, pour les taux de déformation importants (phase d'érosion), par maclage ( $f i g .7 f$ ). On notera que l'acier choisi n'était pas sujet à la transformation martensitique par écrouissage. Sur le $\mathrm{CuAl} 6$, on observe très bien les dislocations qui viennent s'empiler, par bandes, sur les défauts plans comme les joints de grains ou de macles (fig. $7 c$ et $d$ ).

\section{Discussion des résultats}

\subsection{Comparaison de la résistance des matériaux entre eux. Classification}

Si l'on considère, d'un point de vue industriel, que dans la majorité des cas, les pièces sont hors service avant d'avoir atteint la vitesse d'érosion maximale du matériau utilisé [16], le paramètre $T_{i}$ pourrait constituer le critère de comparaison le plus réaliste des matériaux entre eux. Dans le cas de notre étude, il conduit au classement suivant en prenant le cuivre comme métal de référence :

$\begin{array}{ll}\text { Le } \mathrm{CuAl} 2 & \begin{array}{l}\text { est environ } 2 \text { fois plus résistant } \\ \text { que le } \mathrm{Cu} / \mathrm{c} 2\end{array} \\ \text { Le CuZn20 } & \begin{array}{l}\text { est environ } 3 \text { fois plus résistant } \\ \text { que le } \mathrm{Cu} / \mathrm{c} 2 \\ \text { est environ } 5,5 \text { fois plus résistant } \\ \text { que } \mathrm{CuAl} 6\end{array} \\ \text { Le } \mathrm{Z} 2 \mathrm{CND} 17-12 & \begin{array}{l}\text { que environ } 10 \text { fois plus résistant } \\ \text { que le } \mathrm{Cu} / \mathrm{c} 2\end{array}\end{array}$

\subsection{Influence des éléments d'alliage sur l'énergie de défaut d'empilement du cuivre}

L'addition d'éléments d'alliages comme le zinc ou l'aluminium à une matrice constituée de métal pur comme le cuivre à pour effet de faire chuter plus ou moins rapidement son énergie de défaut d'empilement modifiant ainsi son comportement vis-à-vis de la déformation plastique. On enregistre également une augmentation de la dureté initiale (tabl. 2). La variation de $\gamma_{s}$ est fonction, entre autre, de la différence entre le rayon métallique du métal de base et celui de l'élément d'addition.

Ces rayons ont pour valeur $[10]$ :

$\begin{array}{cccc}\text { Métal pur } & \text { Cuivre } & \text { Zinc } & \text { Aluminium } \\ \text { Rayon(A) } & 1,28 & 1,39 & 1,43\end{array}$

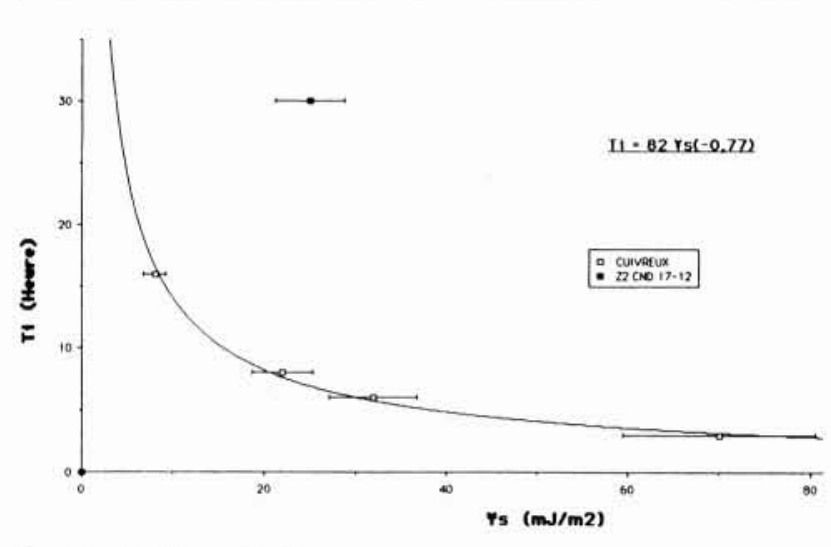

9. Courbe $T_{i}=f\left(\gamma_{s}\right)$.

\subsection{Influence de l'énergie de défaut d'empilement sur la résistance à l'érosion de cavitation}

Les modes de déformation observés sont en accord avec l'énergie de défaut d'empilement des matériaux.

On observe qu'au sein d'une famille d'alliages ayant le même métal de base telle que les cuivreux, une diminution de $\gamma_{s}$ entraîne une augmentation de la résistance à l'érosion de cavitation (fig. $3 a$ et $b$ ). Si l'on trace la courbe $T_{i}=f\left(\gamma_{s}\right)$ (fig. 9), on remarque que la période d'incubation évolue en fonction de $\gamma_{s}$ suivant une courbe logarithmique d'équation :

$$
T_{i}=82 * \gamma_{s}^{-0,77} .
$$

On notera toutefois que la corrélation ne s'applique pas à l'acier austénitique inoxydable. Cette loi ne semble donc pas pouvoir se généraliser à l'ensemble des métaux cubiques à faces centrées.

\subsection{Influence du mode de déformation sur le faciès des cavités}

La différence de faciès observée à l'intérieur des cavités entre le $\mathrm{Cu} / \mathrm{c} 2$ et le $\mathrm{CuAl} 2$ d'une part, et le $\mathrm{CuAl} 6$ d'autre part peut s'expliquer en terme de mode de déformation. En effet, dans le cas des métaux à haute énergie de défaut d'empilement $\left(\gamma_{s}\right)$, le glissement dévié étant favorisé, on observe des lignes et des stries sinueuses tandis que pour les métaux à faible $\gamma_{s}$, la déformation procède par glissements planaires et les stries observées sont beaucoup plus parallèles.

\subsection{Comportement des matériaux vis-à-vis de l'écrouissage. Influence de $\gamma_{s}$}

Les courbes de microdureté mettent en évidence un écrouissage des 5 matériaux étudiés sous l'effet de l'impactage par cavitation.

Le taux d'écrouissage varie entre 2,2 et 2,8 et ne semble pas dépendre de $\gamma_{s}$ puisque les 2 valeurs extrêmes sont d'une part le $\mathrm{Cu} / \mathrm{c} 2(2,19)$ et d'autre part le $\mathrm{CuAl} 2$ $(2,84)$. La valeur moyenne obtenue sur les cinq métaux et alliages est très exactement égale à 2,5. 
Par contre, l'analyse des colonnes 5 et 6 du tableau 4 permet de constater que les métaux et alliages à forte $\gamma_{s}$ qui se déforment par glissement dévié s'écrouissent 10 à 20 fois plus rapidement et sur une profondeur 2 fois plus importante que les métaux de même structure cristallographique, pour lesquels les dislocations vis ne peuvent changer de plan de glissement $\left(\gamma_{s}\right.$ faible $<30 \mathrm{~mJ} \mathrm{~m}^{-2}$ ).

Or, d'après RAO et al. [11] ainsi que BRUNTON et THOMAs [12], la destruction du métal intervient lorsque sa capacité d'écrouissage est arrivée à saturation. Il paraît donc logique que les matériaux à forte $\gamma_{s}$ résistent moins bien à la cavitation que ceux ayant une $\gamma_{s}$ plus faible.

Ces résultats sont d'ailleurs en accord avec les conclusions de SAXENA et ANTOLOVICH [13] concernant l'écrouissage et la propagation des fissures sous sollicitation de fatigue de type oligocyclique sur le cuivre pur et les alliages de cupro-aluminium monophasés.

\subsection{Mécanisme de fragmentation des grains}

L'observation de la propagation des fissures et cratères de cavitation conduit à proposer un mécanisme de convergence des cavités schématisé de la manière suivante :

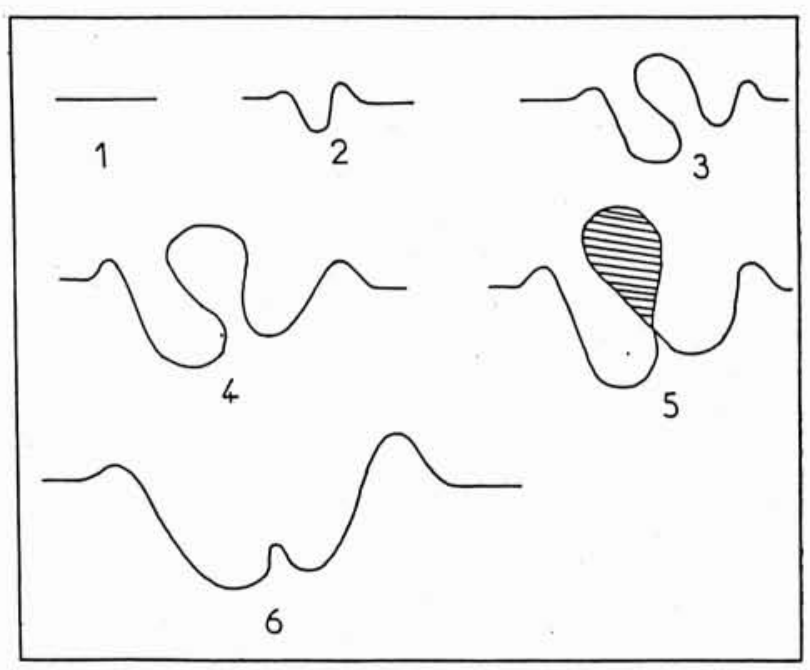

Ce mécanisme constitue un des modes privilégiés d'érosion des matériaux par cavitation.

\section{Conclusion}

Il ressort de cette étude que :

- Le phénomène d'érosion par cavitation est essentiellement un phénomène d'endommagement mécanique.

- Les courbes d'érosion $\Delta v=f(t)$ et $V=g(t)$ permettent, pour chaque matériau, de cerner sa résistance à l'érosion de cavitation, de déterminer ses paramètres caractéristiques $T_{i}$ et $V_{m}$, enfin de comparer les matériaux entre eux.
- Le classement par ordre croissant de résistance à la cavitation des matériaux testés est le suivant :

$\mathrm{Cu} / \mathrm{c} 2<\mathrm{CuAl} 2<\mathrm{CuZn} 20<\mathrm{CuAl} 6<\mathrm{Z} 2$ CND 17-12

- Dans le cas des métaux et alliages de structure C.F.C. testés sur un système du type veine tourbillon, les paramètres $T_{i}$ et $V_{m}$ vérifient l'équation :

$$
V_{m}=0,29 * T_{i}^{-1,167} \text {. }
$$

- Les modes de déformation observés sur chacun des matériaux sont ceux attendus compte tenu de la valeur de leur énergie de défaut d'empilement respective.

Ces modes de déformation submicroscopiques sont ceux également rencontrés lorsque ces mêmes matériaux sont soumis à un endommagement de fatigue oligocyclique [13, 14].

- Pour les matériaux de structure C.F.C., on observe qu'en règle générale, ceux à basse énergie de défaut d'empilement $\left(\gamma_{s}<30 \mathrm{~mJ} \mathrm{~m}^{-2}\right)$ sont plus résistants à l'érosion de cavitation que ceux pour lesquels $\gamma_{s}>$ $30 \mathrm{~mJ} \mathrm{~m}^{-2}$.

Cette remarque est également valable en fatigue oligocyclique et en mécanique de la rupture (vitesse de fissuration) $[13,14,15]$.

- Pour la catégorie spécifique des métaux et alliages cuivreux monophasés, nous avons mis en évidence la relation suivante entre $T_{i}$ et $\gamma_{s}$ :

$$
T_{i}=82 * \gamma_{s}^{-0,77} \text {. }
$$

Cette équation corrobore bien la remarque précédente.

- Il semble que la capacité d'un matériau métallique de structure C.F.C. à résister à l'érosion de cavitation soit liée à :

* Son mode de déformation et notamment sa propension au glissement dévié.

* Sa capacité d'écrouissage.

* Sa vitesse d'écrouissage.

- L'endommagement par érosion de cavitation peut se résumer par le tableau suivant :

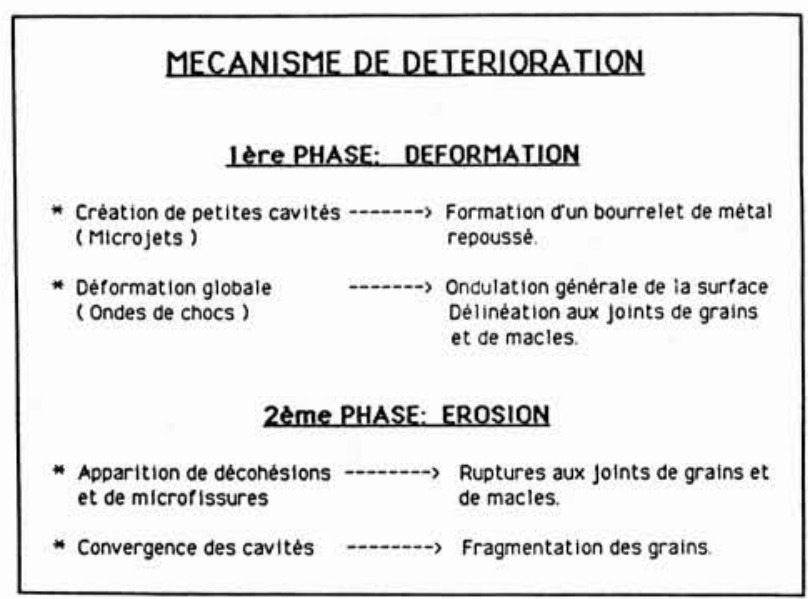




\section{Perspectives}

Compte tenu des rapprochements effectués lors de la discussion des résultats et de la conclusion entre l'endommagement par cavitation et celui par fatigue oligocyclique, il apparaît opportun de confirmer et de poursuivre les investigations dans ce domaine. Dans cette optique, il serait souhaitable de rechercher dans quelle mesure le coefficient d'écrouissage cyclique $n^{\prime}$, issue de la loi de comportement en fatigue plastique :

$$
\sigma_{s}=K^{\prime}\left(\Delta \varepsilon_{p} / 2\right)^{n^{\prime}}
$$

pourrait s'insérer dans un modèle rendant compte de l'endommagement par érosion de cavitation.

$\sigma_{s} \quad$ : Contrainte maximale du cycle stabilisé.

$K^{\prime} \quad$ : Coefficient de ductilité en fatigue.

$\Delta\left(t_{p}\right)$ : Amplitude de la déformation plastique totale.

Le tableau suivant résume les directions dans lesquelles s'orientent les recherches ainsi que les paramètres caractéristiques de chaque voie à explorer.

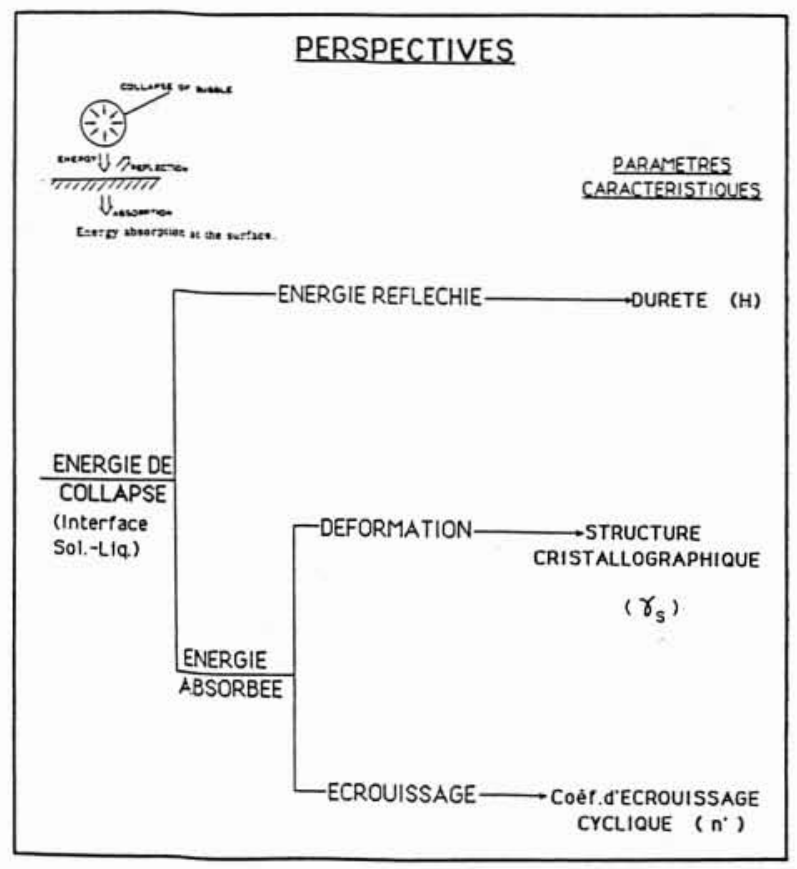

\section{Bibliographie}

[1] Y. Lecoffre, J. Marcoz, R. Anselme, Rapport Neyrtec R20 246, déc. 1980.

[2] P. C. J. Gallagher, Met. Trans., vol. 1, p. 2429, sept. 1970.

[3] C. B. Carter, I. L. F. Ray, Phil. Mag., vol. 35, pp. $189-$ 200, 1977.

[4] L. E. MURR, Shock waves and high strain rate phenomena in metals, Ed. M. A. MEYER and L. E. MURR, Plenum Press N. Y., chap. 37, p. 658, 1981.

[5] A. V. Reddy, G. Sundarajan, R. Sivakumar, P. V. RaO, Acta Met., vol. 32, n 9, pp. 1305-1316, 1984.

[6] I. L. Dillamore, R. E. Smallman, W. T. Roberts, Phil. Mag., vol. 9, p. 517, 1964.

[7] A. Korbel, L. Blaz, H. Dybiec, Met. Technology, pp. 391-397, oct. 1979.

[8] B. J. Thомаs, Métaux corrosion industrie, n 531, p. 363, nov. 1969.

[9] R. E. Schramm, R. P. Reed, Met. Trans. A, vol. 6A, p. 1345 , juill. 1975 .

[10] M. VAn Meersche, J. Feneau-Dupont, Introduction à la cristallographie et à la chimie structurale, Ed. OYEZ, $2^{e}$ éd., p. $117,1976$.

[11] P. V. RaO, C. S. Martin, B. C. S. RaO, N. S. L. RaO, J.T.E.V.A., ASTM, vol. 9, n`3, pp. 189-197, mai 1981.

[12] G. Brunton, J. H. Thomas, Proc. Roy. Soc., A 314, pp. 549-565, 1970.

[13] A. Saxena, D. Antolovich, Met. Trans. A, vol. 6A, pp. 1975-1809, sept. 1975.

[14] J. C. Bathias, J. P. Baillon, La fatigue des métaux et des structures, Ed. Maloine S.A. Paris, 1980.

[15] G. Chalant, L. RemY, ARMINES, Rapport DGRST $n^{\circ}$ 78-7-2442, juill. 1980.

[16] C. M. Preece, I. L. H. Hansson, A Metallurgical Approach to Cavitation Erosion, 6th Int. Conf. on erosion by liquid and solid impacts, Farnborough U.K., 1983, Ed. J. E. Field and N. S. CARneY.

Adresse de l'auteur :

Monsieur J. L. Heuzé

STCAN

Groupe matériaux et structures navales 8, Bd Victor

75015 Paris

Tél. : 40591743 
M. CHIEM : Je remercie M. HeuzÉ pour son exposé si documenté.

M. Colas. (CEA) : Quelqu'un a-t-il fait des expérimentations sur les carbures?

M. HEUZÉ: Il y a beaucoup de références sur les stellites.

M. AVELLAN : Nous avons essayé des douilles en carbure sur le distributeur de la veine tourbillon. Les résultats ont été catastrophiques quant à leur résistance à l'érosion.

M. KARIMI: Des essais de carbure ont été faits en diffẻrents endroits. On a trouvé, dans certains cas des résistances intéressantes pour des alliages à base de nickel et de cobalt.

Que signifient les 4 stades de vos courbes de perte de masse?

M. HEUZÉ : En fait, le $3^{e}$ stade est court et n'apparaît que sur des matériaux ductiles. La durée de la période d'incubation est très faible, mais elle existe. Globalement, ce qui ressort, c'est la partie linéaire qui est caractéristique du matériau. Ce domaine correspond, à mon avis à la résistance du matériau écroui.

M. KARIMI: Vous avez attribué la baisse des valeurs de microduretés en surface à la recristallisation dynamique. $\mathrm{Si}$ on admet que la recristallisation dynamique se produise, le matériau est en permanence écroui et, de ce fait, sa dureté n'est pas forcément diminuée.

$M$. HEUZE : Il y a recristallisation dynamique dès qu'on arrête l'essai. Or, il y a toujours un certain délai entre l'arrêt de l'essai et l'observation des échantillons.

Suit une discussion sur la précision des mesures.

M. KARIMI: Il y a des méthodes très modernes, qui tendent à montrer que les résultats ne s'appliquent peut être pas aux mécanismes avec vitesse de déformation élevée.

M. HEUZÉ: On trouve plus de résultats pour les matériaux à faible énergie de défaut d'empilement.

M. KARIMI : Il faut faire attention aux tendances trop générales et aux relations liant l'érosion aux microduretés ou à l'énergie de défaut d'empilement. Ce genre de corrélation semble être confirmé pour la même famille des alliages, mais d'une catégorie des matériaux à l'autre, il n'a pas été vérifié expérimentalement.

M. HEUZÉ: Il y a une bonne corrélation pour les alliages cuivreux. Lorsqu'on veut l'étendre aux matériaux de structure cubique à face centrée, cela ne marche plus. Quant aux autres structures, il reste bien des points d'interrogation.
M. MAAMOURI: Quelle est la charge utilisée pour mesurer la microdureté ? (Réponse : $490,3 \mathrm{mN}$.)

La dureté varie en fonction de la charge utilisée, en particulier pour les matériaux fortement écrouis. Il faut, pour chaque échantillon, faire une courbe jusqu'à ce que l'on obtienne une constante.

M. HEUZÉ : Pour l'échantillon ayant subi 1 million d'impacts, on constate une décroissance de la microdureté. Cela m'a surpris ainsi que beaucoup de gens. Il y a des preuves concordantes qui indiquent qu'il y a restauration. On observe une augmentation de la taille des cellules pour cet échantillon, ce qui indique qu'il y a diminution de la densité des dislocations. Les essais de diffraction montrent un élargissement de la largeur intégrale du pic. Une fois passée la période d'incubation, correspondant au maximum d'écrouissage, on a obtenu une décroissance de la dureté, notamment pour 1 million de chocs.

Ces trois observations permettent de faire l'hypothèse d'une recristallisation.

M. MAAMOURI : Il faut faire attention aux tailles des empreintes par rapport à celles des cellules et respecter une certaine distance entre deux empreintes.

M. CHIEM : Ce qui est important, c'est le rapport entre la taille des cellules et l'épaisseur de la paroi des cellules. La courbe de dureté passe par un maximum puis il y a libération des contraintes et les cellules s'agrandissent de nouveau. Le rapport entre la taille des cellules et l'épaisseur de la paroi peut atteindre 7 à 9 pour le cuivre.

M. MAAMOURI : Le mâclage peut aussi provoquer une augmentation de la dureté

M. CHIEM : C'est normal, car le mâclage peut entraîner un durcissement.

M. HEUZÉ : Mes conditions hydrodynamiques étaient telles que je restais au stade de la déformation par formation de cellules tridimensionnelles, et par variation de leur diamètre. J'obtenais une recristallisation.

M. FARHAT: N'y a-t-il pas un effet du gaz dissous? Y a-t-il un moyen de contrôle?

M. HEUZÉ : Oui : On mesure l'oxygène dissout par un système Orbisphère. On procédait avant par des dégazages réguliers. C'est vrai que la teneur en air de l'eau est très importante.

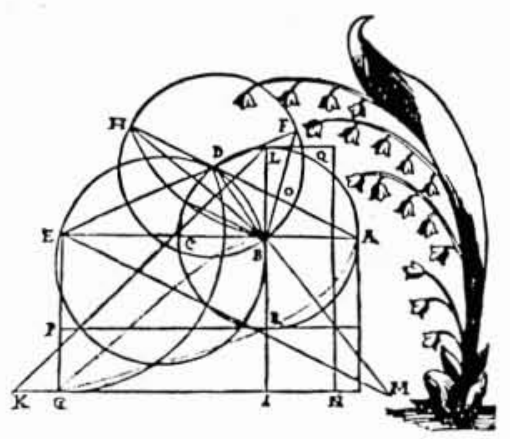

